\title{
AN ENTIRE TRANSCENDENTAL FUNCTION WHOSE INVERSE TAKES SETS OF FINITE MEASURE INTO SETS OF FINITE MEASURE
}

\author{
BY W. J. SCHNEIDER
}

\author{
Communicated by W. Fuchs, April 25, 1966
}

In a recent issue of this journal [2] the following research problem was posed by F. Gross:

Let $S$ be an arbitrary region of finite measure. ${ }^{1}$ Does there exist a transcendental meromorphic function with the property that the pre-image $f^{-1}(S)$ is of finite measure?

The following theorem answers the above question not just for meromorphic functions but for entire functions also.

THEOREM. There exists an entire transcendental function $f(z)$ whose inverse takes sets of finite measure into sets of finite measure.

Proof. Let $D=\left\{z=x+i y|x>0| y \mid,<1 /\left(1+x^{2}\right)\right\}$ and let $E$ be the complement in the plane of the closure of $D$. Also let $c(z)$ be a conformal map from the outside of $|z-1|=\epsilon(\epsilon$ a small positive constant to be determined later) into the unit disk which takes the point at infinity into the origin. By a particular case of Runge's theorem [3] and Rouche's theorem there exists a rational function $R_{1}(z)$ with the following properties:

(i) $R_{1}(z)$ has a pole only at $z=2$.

(ii) $R_{1}(z)$ so closely approximates $c(z)$ on $E$ that $R_{1}(z)$ on $E$ has the following properties:

(a) measure $R_{1}(E)$ less than one.

(b) $R_{1}(z)+z$ is $1-1$ (this is always possible by choosing $\epsilon$ small enough) on $E$.

(c) $\lambda=\left|R_{1}(-1)-R(-2)\right| \neq 0$.

By the same argument there exists a rational function $R_{2}(z)$ which has the following properties:

(i) $R_{2}(z)$ has a pole only at $z=3$.

(ii) $R_{2}(z)$ so closely approximates $R_{1}(z)$ in $E \cup G_{3}(=\{z=x+i y \mid x$ $<3 / 2\}$ ) that $R_{2}(z)$ on $E \cup G_{3}$ has the following properties:

(a) measure $R_{2}(E)$ less than one.

(b) $R_{2}(z)+z$ is $1-1$ on $E$.

(c) $\left|R_{2}(z)-R_{1}(z)\right|<\lambda / 8$ on $E \cup G_{3}$.

Continuing inductively there exists a rational function $R_{n}(z)$ which has the following properties:

${ }^{1}$ Dr. Gross was kind enough to point out to me that in the statement of the problem accidentally the words "of finite measure" were left out. 
(i) $R_{n}(z)$ has a pole only at $z=n+1$.

(ii) $R_{n}(z)$ so closely approximates $R_{n-1}(z)$ in $E \cup G_{2 n-1}$ ( $=\{z=x+i y \mid x<(2 n-1) / 2\}$ ) that $R_{n}(z)$ on $E \cup G_{2 n-1}$ has the following properties:

(a) measure $R_{n}(E)$ less than one.

(b) $R_{n}(z)+z$ is $1-1$ on $E$.

(c) $\left|R_{n}(z)-R_{n-1}(z)\right|<\lambda / 2^{n+1}$ on $E \cup G_{2 n-1}$.

By a theorem of Ostrowski [1] on the convergence of meromorphic functions in the chordal metric $\lim _{n \rightarrow \infty} R_{n}(z)=h(z)$ exists and is meromorphic in the plane. However by the way we choose the poles of $R_{n}(z)$ to converge to infinity $h(z)$ is clearly analytic. Since measure of $h(E)$ is less than or equal to one $h(z)$ cannot be a nonconstant polynomial and $h(z)$ cannot be constant since $|h(-1)-h(-2)| \geqq \lambda / 2$. Now let $f(z)=h(z)+z$ and let $S$ be any set of finite measure in the plane. The part of $f^{-1}(S)$ contained in $D$ certainly has finite measure. The part of $f^{-1}(S)$ in $E$ (call it $K$ ) must have finite measure, otherwise we would be led to a contradiction since [measure of $f(K)$ ] $\geqq$ [measure of $K]-[$ measure of $h(K)]$ (from $\left|f^{\prime}(z)\right|^{2} \geqq 1-\left|h^{\prime}(z)\right|^{2}$ for all $z$ in $E$ and the fact $f(z)$ has to be $1-1$ on $E$ ) and the measure of $h(K)$ is less than one.

\section{REFERENCES}

1. C. Carathéodory, Theory of functions, Vol. 1, p. 190, Chelsea, New York, 1954.

2. F. Gross, Function theory 18, Bull. Amer. Math. Soc. 71 (1965), 853.

3. S. Saks and A. Zygmund, Analytic functions, p. 177, Monographie, Matematyczne, vol. 28, Warsaw and Wraclaw, 1952.

Syracuse University 